\title{
The Bekenstein-Hawking Corpuscular Cascading from the Back-Reacted Black Hole
}

\author{
Ali Övgün ${ }^{1,2}$ \\ ${ }^{1}$ Instituto de Física, Pontificia Universidad Católica de Valparaíso, Casilla 4950, Valparaíso, Chile \\ ${ }^{2}$ Physics Department, Eastern Mediterranean University, Famagusta, Northern Cyprus, Mersin 10, Turkey
}

Correspondence should be addressed to Ali Övgün; ali.ovgun@pucv.cl

Received 7 March 2017; Revised 6 June 2017; Accepted 5 July 2017; Published 20 August 2017

Academic Editor: George Siopsis

Copyright (c) 2017 Ali Övgün. This is an open access article distributed under the Creative Commons Attribution License, which permits unrestricted use, distribution, and reproduction in any medium, provided the original work is properly cited. The publication of this article was funded by $\mathrm{SCOAP}^{3}$.

Exciting peculiarities of Planck-scale physics have immediate effects on the Bekenstein-Hawking radiation emitted from black holes (BHs). In this paper, using the tunneling formalism, we determine the Bekenstein-Hawking temperature for the vector particles from a back-reacted black hole (BBH) constructed from a conformal scalar field surrounded by a BTZ (Banados-Teitelboim-Zanelli) $\mathrm{BH}$. Then, under the effect of the generalized uncertainty principle, we extend our calculations for scalar particles to understand the effects of quantum gravity. Then, we calculate an evaporation time for the $\mathrm{BBH}$, the total number of Bekenstein-Hawking particles, and the quantum corrections of the number. We observe that remnants of the $\mathrm{BH}$ evaporation occur and that they affect the Bekenstein-Hawking temperature of the $\mathrm{BBH}$ as well as the total number of Bekenstein-Hawking particles.

\section{Introduction}

Many years ago, Bekenstein suggested that, in a quantum theory of gravity, the surface area of a black hole (BH) should have a discrete spectrum [1]. In 1972, Bekenstein wrote a seminal paper showing that the entropy of a $\mathrm{BH}$ is exactly proportional to the size of its event horizon; then, he showed that there is a maximum amount of information that can be stored in a finite region of space, a concept known as the Bekenstein bound $[2,3]$. Following Bekenstein's insights, some of the most significant ideas in theoretical physics were born, such as Bekenstein-Hawking radiation [2-5], the $\mathrm{BH}$ information paradox, the holographic principle, and the $\mathrm{BH}$ firewall paradox. It is a remarkable fact that, according to the seminal works of Bekenstein and then Hawking [2-5], BHs are not entirely black. That surprising claim was made over forty years ago. Examining the behavior of quantum fluctuations around the event horizon of a $\mathrm{BH}$, Bekenstein and Hawking substantiated the theory that $\mathrm{BHs}$ emit thermal radiation with a constant temperature (the so-called Bekenstein-Hawking temperature) that is directly proportional to the surface gravity $\kappa$, which is the gravitational acceleration experienced at the BH's horizon $[2,3,5]$ :

$$
T_{H}=\frac{\hbar \kappa}{2 \pi} .
$$

When BHs evaporate, their temperatures increase adiabatically as a function of their remaining mass. Quantum fluctuations create a virtual particle pair near the BH horizon. While the particle with negative energy tunnels into the horizon (absorption), the other having positive energy flies off into spatial infinity (emission) and produces BekensteinHawking radiation (BHR). There are various methods for calculating the level of BHR, and the two most popular tunneling methods are the null geodesic method and the Hamilton-Jacobi (HJ) method [6-39]. Both approaches use the tunneling method by applying the WKB (WentzelKramers-Brillouin) approximation and finding the emission and absorption probabilities of the tunneling particles, which give the tunneling rate $\Gamma$ as follows [40-51]:

$$
\Gamma=\frac{P_{\text {emission }}}{P_{\text {absorption }}}=e^{-2 \operatorname{Im} S}=e^{-E / T},
$$


where $S$ is the action of the classically forbidden trajectory of the tunneling particle, which has an energy $E$ and a temperature $T$. Here, the conservation of energy plays an important role. First, there is a transition between states with the same total energy; then, it radiates when the mass of the residual hole must go down. Also, there is a way to lower the mass of the $\mathrm{BH}$. This brings us to the idea that $\mathrm{BH}$ are thought to be excited states in quantum gravity, so that one can derive the $\mathrm{BH}$ temperature, which is related to the Boltzmann factor, using the imaginary part of the action during the process of the emission of an s-wave from the inside to the outside of the horizon [6-11]. For calculations of the tunneling probability, it is simple to use a WKB approximation. It should be noted that one can ignore the higher-order terms (and use only a linear-order expansion), which have a selfinteraction effect that results from the conservation of energy [18-21]. One of the methods for finding $S$ is the HJ method. This method is generally employed by substituting a suitable ansatz, with consideration of the symmetries of the spacetime, into the relativistic $\mathrm{HJ}$ equation [23, 24, 38-40, 52, 53]. The resulting radial integral always possesses a pole located at the event horizon. However, using the residue theory, the associated pole can be analytically avoided. It is argued that $\mathrm{BHs}$ emit radiation that is dissimilar to emitted radiation from a thermal objects except that the $\mathrm{BH}$ radiation spectrum is semiclassically sparse. At least in the weak regime, a quantum $\mathrm{BH}$ retains a discrete profile that may result from the simplicity of the spectrum. Different complicated spectra can be obtained, and they cause the radiation to look continuous enough in profile to use different quantum theories [54]. Furthermore, from the emission spectrum of the $\mathrm{BH}$ radiation, if the grey body factor is ignored, semiclassical treatment shows that $\mathrm{BHs}$ radiate and that the resulting emission spectrum is similar to that of the thermal radiation of a blackbody. Reference [55] showed that there is a very long time gap between the emissions; moreover, the same conclusion that the cascade of BHR is very sparse was also shown. For an observer at the asymptotic infinity, the flux of BHR from a BH is intensely sparse [56], especially for a $3+1$ Schwarzschild $\mathrm{BH}$. However, for dimensional cases that are lower/higher than $3+1$ dimensions, Bekenstein-Hawking cascades have a continuous character $[1,57-60]$.

The collapsed pure initial state supports the formation of a $\mathrm{BH}$, which was concluded by Hawking, and then the radiation shades into a high-entropy mixed state. This is contrary to the fundamentals of quantum mechanics which cannot maintain its pure state, so it becomes mixed up and causes an information paradox [59]. Here, we use a back-reacted black hole (BBH), which is a BTZ (Banados-Teitelboim-Zanelli) BH surrounded by a conformal scalar field [54, 61-64], to investigate the BHR from tunneling vector particles and quantum gravity-affected scalar particles. Our motivation for working on $2+1$ dimensions is to make the problem much easier. First, there are no propagating degrees of freedom. Quantum gravity in $2+1$ dimensions is renormalizable and finite. It is also well known that the findings in $2+1$ dimensions are a good guide to what would happen in the real world. We will now find out what happens to the BekensteinHawking temperature of scalar and vector particles from a $\mathrm{BBH}$.

This paper is organized as follows. In Section 2, we introduce the geometric and thermodynamic features of $\mathrm{BBH}$ space-time. In Section 3, we study the Proca equation to find a massive boson in this geometry. Then, we employ the HJ method with the separation of variables technique to obtain the BHR level of the BBH. Then, in Section 4, we repeat the calculations for the radiating scalar particles under the effect of quantum gravity. We compute the corrected BekensteinHawking temperature. Finally, in Section 5, we obtain the total number of outgoing Bekenstein-Hawking particles, and the paper ends with our conclusions in Section 6.

\section{Back-Reacted Black Hole}

The $\mathrm{BBH}$ is constructed from a BTZ BH surrounded by a conformal scalar field. For this reason, one calculates the stress-energy tensor $\left\langle T_{\mu \nu}\right\rangle$ by considering the transparent boundary conditions at infinity; the approximate solution coincides, on a finite domain, with the exact solution [54, 62, 63]. Transparent boundary conditions, whose name comes from the fact that they are designed to be transparent to outgoing perturbations, have the attribute of being nonreflecting. Transparent boundary conditions were originally designed for the Schrodinger equations but have also been applied to the wave equation [65] and the conformal scalar field $[54,62,63]$. The semiclassical equations are used to find the $O(\hbar)$ correction to the $\mathrm{BBH}$ geometry.

$$
G_{\mu \nu}+\Lambda g_{\mu \nu}=\kappa\left\langle T_{\mu \nu}\right\rangle \text {. }
$$

The exact solution of the metric of the $\mathrm{BBH}$ in the presence of a conformally coupled scalar field in three dimensions can be expressed as [54]:

$$
d s^{2}=-f(r) d t^{2}+f^{-1}(r) d r^{2}+r^{2} d \varphi^{2},
$$

where

$$
f(r)=\left(\frac{r^{2}}{l^{2}}-M-\frac{2 l_{p} F(M)}{r}\right) .
$$

It is noted that $l^{-2}$ is the cosmological constant, $M$ is the mass of the $\mathrm{BBH}$, and $l_{p}$ is the length of the Planck. Note that for transparent boundary conditions $F(M)$ is defined as follows [54]:

$$
F(M)=\frac{M^{3 / 2}}{2 \sqrt{2}} \sum_{n=1}^{\infty} e^{-i n \delta} \frac{\cosh [2 \pi n \sqrt{M}]+3}{(\cosh [2 \pi n \sqrt{M}]-1)^{3 / 2}},
$$

where $\delta$ is an arbitrary phase. For a field with periodic boundary conditions $\delta=0$ (bosons), while for fields with antiperiodic boundary conditions $\delta=\pi$ (fermions). 
The BTZ BH is recovered at $F(M)=0$ for $M \gg$ 1. Furthermore, the metric has an event horizon which is located at

$$
\begin{aligned}
r_{h} & =\sqrt{M} l+\frac{l_{p} F(M)}{M} \\
T_{H} & =\frac{f^{\prime}\left(r_{h}\right)}{4 \pi}=\left[\frac{\sqrt{M}}{2 \pi l}+\frac{l_{p} F(M)}{\pi M l^{2}}\right] .
\end{aligned}
$$

Since the perturbative expansion has no corrections from graviton loops, only radiative corrections are obtained from quantum excitations of the matter fields, because the quantum gravity in $2+1$ dimensions is renormalizable and finite. Furthermore, it is observed that the temperature is linear in $F(M)$. The corrections are removed when the $F(M) \approx e^{-\pi M}$ for a large $M$, so the back-reaction is more dominant for small masses than for the Planck mass. The other important point is that $\left\langle T_{\mu \nu}\right\rangle$, which depends on the ratio $l_{p} / l$, causes corrections so that, for a small cosmological constant, we can ignore the perturbation of the geometry produced by radiation fields.

\section{Bekenstein-Hawking Radiation of Vector Particles from BBH}

To calculate the BHR of the tunneling vector particles from the $\mathrm{BBH}$, the Proca equation is used on the $\mathrm{BBH}$ geometry. The massive vector particles are described by the Proca equation with the wave function $\psi$ given by [22-27]

$$
\frac{1}{\sqrt{-g}} \partial_{\mu}\left(\sqrt{-g} \psi^{\nu \mu}\right)+\frac{m^{2}}{\hbar^{2}} \psi^{\nu}=0
$$

in which

$$
\psi_{\nu \mu}=\partial_{\nu} \psi_{\mu}-\partial_{\mu} \psi_{\nu}
$$

To leading order in the energy we can neglect the effects of the self-gravitation of the particle [6]. Then we solve the Proca equation on the background of the $\mathrm{BBH}$,

$$
\begin{aligned}
& \frac{f}{r}\left\{\partial_{r}\left[\sqrt{\frac{f r^{2}}{f}}\left(\partial_{t} \psi_{1}-\partial_{r} \psi_{0}\right)\right]\right. \\
& \left.+\partial_{\varphi}\left[\frac{1}{f r}\left(\partial_{t} \psi_{2}-\partial_{\varphi} \psi_{0}\right)\right]\right\}+\frac{m^{2}}{\hbar^{2}} \psi_{0}=0, \\
& \frac{1}{f r}\left\{\partial_{t}\left[\sqrt{\frac{f^{2} r^{2}}{f}}\left(\partial_{t} \psi_{1}-\partial_{r} \psi_{0}\right)\right]\right. \\
& \left.+\partial_{\varphi}\left[\frac{f}{r}\left(\partial_{r} \psi_{2}-\partial_{\varphi} \psi_{1}\right)\right]\right\}+\frac{m^{2}}{\hbar^{2}} \psi_{1}=0,
\end{aligned}
$$

$$
\begin{aligned}
& r\left\{\partial_{t}\left[\frac{1}{f r}\left(\partial_{t} \psi_{2}-\partial_{\varphi} \psi_{0}\right)\right]\right. \\
& \left.\quad+\partial_{r}\left[\sqrt{\frac{f^{2}}{r^{2}}}\left(\partial_{\varphi} \psi_{1}-\partial_{r} \psi_{2}\right)\right]\right\}+\frac{m^{2}}{\hbar^{2}} \psi_{2}=0
\end{aligned}
$$

It is assumed that the solution exists in the form of the vector functions:

$$
\psi_{\nu}=c_{\nu} \exp \left[\frac{i}{\hbar} S(t, r, \varphi)\right]
$$

where $c_{v}=c_{0}, c_{1}, c_{2}$ are some arbitrary constants, with the help of action

$$
\begin{aligned}
S(t, r, \varphi)= & S_{0}(t, r, \varphi)+\hbar S_{1}(t, r, \varphi)+\hbar^{2} S_{2}(t, r, \varphi) \\
& +\cdots .
\end{aligned}
$$

Then the above equations become

$$
\begin{aligned}
& f\left[c_{0}\left(\partial_{r} S_{0}\right)^{2}-c_{1}\left(\partial_{r} S_{0}\right)\left(\partial_{t} S_{0}\right)\right] \\
& +\frac{1}{r^{2}}\left[c_{0}\left(\partial_{\varphi} S_{0}\right)^{2}-c_{2}\left(\partial_{\varphi} S_{0}\right)\left(\partial_{t} S_{0}\right)\right]+m^{2} c_{0}=0 \\
& \frac{1}{f}\left[c_{0}\left(\partial_{r} S_{0}\right)\left(\partial_{t} S_{0}\right)-c_{1}\left(\partial_{t} S_{0}\right)^{2}\right] \\
& +\frac{1}{r^{2}}\left[c_{1}\left(\partial_{\varphi} S_{0}\right)^{2}-c_{2}\left(\partial_{\varphi} S_{0}\right)\left(\partial_{r} S_{0}\right)\right]+m^{2} c_{1}=0 \\
& \frac{1}{f}\left[c_{0}\left(\partial_{\varphi} S_{0}\right)\left(\partial_{t} S_{0}\right)-c_{2}\left(\partial_{t} S_{0}\right)^{2}\right] \\
& \quad+f\left[c_{2}\left(\partial_{r} S_{0}\right)^{2}-c_{1}\left(\partial_{\varphi} S_{0}\right)\left(\partial_{r} S_{0}\right)\right]+m^{2} c_{2}=0 .
\end{aligned}
$$

Using the WKB approximation, the action can be chosen at the leading order in $\hbar$ as

$$
S_{0}(t, r, \theta)=-E t+W(r)+J \varphi+\mathbb{k} .
$$

Here the energy is defined by $E$ and the angular momentum of the spin-1 vector particles is defined by $J$; furthermore $\mathbb{k}$ is a constant. Then we use (14) inside the solutions of (13) considering the leading order in $\hbar$. Then $3 \times 3$ matrix (let us say (:) matrix) is obtained: $:\left(c_{0}, c_{1}, c_{2}\right)^{T}=0$. It is noted that the superscript $T$ means the transition to the transposed vector. So, the nonzero components of the matrix of $(;)$ are calculated as follows:

$$
\begin{aligned}
& \odot_{11}=f\left(\partial_{r} W\right)^{2}+\frac{\left(\partial_{\varphi} J\right)^{2}}{r^{2}}+m^{2}, \\
& \odot_{12}=f E \partial_{r} W \text {, } \\
& \ominus_{13}=\frac{E \partial_{\varphi} J}{r^{2}}, \\
& \ominus_{21}=\frac{E \partial_{r} W}{f} \text {, }
\end{aligned}
$$




$$
\begin{aligned}
& \odot_{22}=\frac{E^{2}}{f}-\frac{\left(\partial_{\varphi} J\right)^{2}}{r^{2}}-m^{2} \\
& \odot_{23}=\frac{\left(\partial_{r} W\right) \partial_{\varphi} J}{r^{2}} \\
& \odot_{31}=\frac{E \partial_{\varphi} J}{f} \\
& \odot_{32}=f\left(\partial_{r} W\right) \partial_{\varphi} J \\
& \ominus_{33}=\frac{E^{2}}{f}-f\left(\partial_{r} W\right)^{2}-m^{2} \text {. }
\end{aligned}
$$

The condition of the finding nontrivial solutions of any linear equations $(\operatorname{det}(;)=0)$ gives

$$
m^{2}\left[f\left(\partial_{r} W\right)^{2}-\left(\frac{E^{2}}{f}-\frac{\left(\partial_{\varphi} J\right)^{2}}{r^{2}}-m^{2}\right)\right]^{2}=0 .
$$

Then the solution of the above equation for the radial function $W(r)$ yields

$$
W_{ \pm}(r)= \pm \int \sqrt{\frac{E^{2}-f\left(m^{2}+\left(\partial_{\varphi} J\right)^{2} / r^{2}\right)}{f^{2}}} d r .
$$

It is noted that $W_{+}(r)$ and $W_{-}(r)$ show that the vector particles move away from the $\mathrm{BBH}$ and move towards to the $\mathrm{BBH}$. Moreover, there are poles located at horizon $r=r_{h}$; the imaginary part of $W_{ \pm}\left(r_{h}\right)$ can be calculated by using the complex path integration method [27, 34, 35]. Then, the integral becomes

$$
\operatorname{Im} W_{ \pm}\left(r_{h}\right)= \pm \frac{\pi}{f^{\prime}\left(r_{h}\right)} E .
$$

Then we obtain the probabilities of the vector particles tunnels through the horizon out/in:

$$
\begin{gathered}
P_{\text {emission }}=e^{-(2 / \hbar) \operatorname{Im} S_{+}}=e^{\left[-(2 / \hbar)\left(\operatorname{Im} W_{+}+\operatorname{Im} \mathbb{k}\right)\right]}, \\
P_{\text {absorption }}=e^{-(2 / \hbar) \operatorname{Im} S_{-}}=e^{\left[-(2 / \hbar)\left(\operatorname{Im} W_{-}+\operatorname{Im} \mathbb{k}\right)\right]} .
\end{gathered}
$$

The ingoing vector particles must have the $P_{\text {absorption }}=1$ which mean that their chance to fall inside is $100 \%$ in agreement with the definition of the $\mathrm{BH}[26,27,34]$. Consequently, we can choose the $\operatorname{Im} \mathbb{k}=-\operatorname{Im} W_{-}$; then it becomes $W_{+}=$ $-W_{-}$and we can calculate the tunneling rate of the vector particles as

$$
\begin{aligned}
\Gamma & =P_{\text {emission }}=\exp \left(-\frac{4}{\hbar} \operatorname{Im} W_{+}\right) \\
& =\exp \left(-\frac{4 \pi}{f^{\prime}\left(r_{h}\right)} E\right) .
\end{aligned}
$$

Note that the Bekenstein-Hawking temperature of the $\mathrm{BBH}$ is recovered by using relation between the tunneling rate and the Boltzmann factor $e^{-E / T}$. Hence, the Bekenstein-Hawking temperature of $\mathrm{BBH}$ is $[54,62,63]$

$$
T_{H}=\left[\frac{\sqrt{M}}{2 \pi l}+\frac{l_{p} F(M)}{\pi M l^{2}}\right] .
$$

Hence, we obtain the correct Bekenstein-Hawking temperature of the $\mathrm{BBH}$ using the tunneling method for the spin-1 particles.

\section{Bekenstein-Hawking Radiation of Scalar Particles from BBH with the Effect of the Quantum Gravity}

In this section, we check the effects of quantum gravity on the BHR of scalar particles from a BBH using the generalized uncertainty principle (GUP). One may ask how we can extend the quantum mechanics, considering the gravitational interactions. The answer is a quantum theory of gravity, which is the biggest problem in theoretical physics. One common feature among various quantum gravity theories, such as string theory, loop quantum gravity, and noncommutative geometry, is the existence of a minimum measurable length [66-74]. An effective model for realizing the minimum length is the GUP, based on which the first generalized uncertainty relationship was proposed by [68], to solve the problem of quantum gravity.

First, we solve the Klein-Gordon (KG) equation under the effect of the GUP on the background of the BBH to find the BHR. The commutation relationship is modified by using quantum gravity as follows [45, 67-69]:

$$
\left[x_{i}, p_{j}\right]=i \hbar\left(1+\alpha^{2} p^{2}\right) \delta_{i j}
$$

and the GUP is derived as follows:

$$
\Delta x \Delta p \geq \frac{\hbar}{2}\left\{1+\alpha^{2}(\Delta p)^{2}\right\}
$$

It is noted that $\alpha=\alpha_{0} /\left(m_{p}^{2}\right)=l_{p}^{2} / \hbar^{2}$ is a small value, $m_{p}=$ $\hbar / l_{p}$ is the Planck mass, $l_{p}$ is the Planck length $\left(\sim 10^{-35} \mathrm{~m}\right)$, and $\alpha_{0}<10^{34}$ is a dimensionless parameter.

Quantum gravity effects the KG equation which is the relativistic wave equation for the scalar particles, because the position, momentum, and energy operators are modified due to the GUP, respectively, as follows:

$$
\begin{aligned}
x_{i} & =x_{o i}, \\
p_{i} & =p_{0 i}\left(1+\alpha^{2} p^{2}\right), \\
\varepsilon & =E\left(1+\alpha^{2} E^{2}\right) .
\end{aligned}
$$

Furthermore, the frequency is also generalized as

$$
\bar{\omega}=E\left(1-\alpha E^{2}\right)
$$


with the energy operator $E=i \hbar \partial_{0}$. One can calculate the square of momentum operators up to order $\alpha^{2}$ as

$$
\begin{aligned}
p^{2} & =-\hbar^{2}\left[1-\alpha^{2} \hbar^{2} \partial_{j} \partial^{j}\right] \partial_{i}\left[1-\alpha^{2} \hbar^{2} \partial_{j} \partial^{j}\right] \partial^{i} \\
& =-\hbar^{2}\left[\partial_{i} \partial^{i}-2 \alpha^{2} \hbar^{2}\left(\partial_{j} \partial^{j}\right)\left(\partial_{k} \partial^{k}\right)\right]+\left(\alpha^{4}\right),
\end{aligned}
$$

where, in the last step, we only keep the leading order term of $\alpha$.

Therefore, the generalized KG equation with the wave function $\Psi$ can be written as [45]

$$
\begin{aligned}
- & (i \hbar)^{2} \partial^{t} \partial_{t} \Psi \\
& =\left[(i \hbar)^{2} \partial^{i} \partial_{i}+m_{p}^{2}\right]\left[1-2 \alpha\left((i \hbar)^{2} \partial^{i} \partial_{i}+m_{p}^{2}\right)\right] \Psi .
\end{aligned}
$$

Herein, we substitute the ansatz for the semiclassical wave function $\Psi$ of the scalar particles

$$
\Psi=C e^{(i / \hbar) S(t, r, \theta)},
$$

where $C$ is the constant, into the generalized KG equation (27) with the BBH metric (4) which is the background of scalar particle motion. Then, the differential equation for the action $S$ is calculated as follows:

$$
\begin{aligned}
& \frac{1}{f}\left[\left(d_{t} S\right)^{2}=f\left(d_{r} S\right)^{2}+\frac{1}{r^{2}}\left(d_{\theta} S\right)^{2}+m_{p}^{2}\right] \\
& \quad \times\left[1-2 \alpha\left(f\left(d_{r} S\right)^{2}+\frac{1}{r^{2}}\left(d_{\theta} S\right)^{2}+m_{p}^{2}\right)\right] .
\end{aligned}
$$

The separation of variables is used to solve the generalized KG equation after (29) is expanded into the lowest order of $\hbar$

$$
S(t, r, \theta, \varphi)=-E t+W(r)+j(\theta)+\varrho,
$$

where $\varrho$ is the constant. Then we substitute (30) into (29) to solve for the $W(r)$. Then the radial part of the scalar wave function is found that

$$
W \pm=\int d r \frac{1}{f} \frac{\sqrt{E^{2}-m_{p}^{2}\left(1-2 \alpha m_{p}^{2}\right) f}}{\sqrt{1-2 \alpha m_{p}^{2}}},
$$

where the positive and negative \pm signatures are for the outgoing and ingoing scalar particles. To solve this integral, after using the residue method around the pole at the horizon, we obtain the solution

$$
W \pm=i \pi \frac{E}{f^{\prime}\left(r_{h}\right) \sqrt{1-2 \alpha m_{p}^{2}}} .
$$

Herein, similarly to the previous section, we use the fact of the probability of ingoing particles to $100 \%\left(P_{\text {absorption }}=1\right)$. Thus, the tunneling rate is calculated for the scalar particles with the effect of the quantum gravity as

$$
\begin{aligned}
\Gamma & =P_{\text {emission } / \text { absorption }}=e^{-(4 / \hbar) \operatorname{Im} W_{+}} \\
& =e^{-\left(4 \pi i / f^{\prime}\left(r_{h}\right) \sqrt{1-2 \alpha m_{p}^{2}}\right) E} .
\end{aligned}
$$

Now, it is easy to recover Bekenstein-Hawking temperature for the scalar particles with the effect of quantum gravity

$$
\begin{aligned}
T_{H} & =\frac{f^{\prime}\left(r_{h}\right) \sqrt{1-2 \alpha m_{p}^{2}}}{4 \pi} \\
T_{H} & =\left[\frac{\sqrt{M}}{2 \pi l}+\frac{l_{p} F(M)}{\pi M l^{2}}\right] \sqrt{1-2 \alpha m_{p}^{2}} .
\end{aligned}
$$

It is easily observed that when we choose $\alpha=0$, it is equal to the original result of Bekenstein-Hawking temperature. Hence, the BHR of the BBH with the effect of the quantum gravity has remnants.

\section{Total Number of Tunneling Massless Bekenstein-Hawking Particles}

In this section, we calculate the estimation of the total number of massless quanta emitted by the BBH. One shows that the total number of quanta emitted by the $\mathrm{BBH}$ is proportional to the square of the BBH's initial mass in Planck units. Firstly we introduce Planck's law of black body radiation for two space dimensions to calculate the spectral luminosity density of an ideal black body as follows $\left(8 \pi^{2}=\hbar=G=k_{B}=c=1\right)$ [75-77]:

$$
d L=\frac{\omega^{2}}{\exp \omega / T-1} d \omega d A .
$$

Note that $\omega, A$, and $T$ are the energy, surface area, and the temperature, respectively. The result of the integration of (35) is the Stefan-Boltzmann law which is stated that the power emitted per unit area of the surface of a black hole is directly proportional to the 4th power of its temperature $[75,76]$. After we take integral of (35), for two space dimensions, the luminosity is found as

$$
L \approx A T^{3} .
$$

Then the emission rate of the emitted quanta is obtained as

$$
\Gamma=\frac{1}{\omega} \frac{d L}{d \omega}=\int \frac{g A \omega^{2} d \omega}{\exp \omega / T-1},
$$

where $g$ is the number of radiating degrees of freedom,

$$
\Gamma \approx A T^{2} .
$$

Now, we recall the Bekenstein-Hawking temperature of the $\mathrm{BBH}$ and the area of the $\mathrm{BBH}$ in $2+1$ dimension as

$$
\begin{gathered}
T \approx \frac{\sqrt{M}}{l}+\frac{l_{p} F(M)}{M l^{2}}, \\
A \approx r_{h} \approx\left[\sqrt{M} l+\frac{l_{p} F(M)}{M}\right] .
\end{gathered}
$$

One shows that the mass loss rate is related to the luminosity as follows:

$$
\frac{d M}{d t}=-L=-\frac{M^{2}}{l^{2}}
$$


Then the evaporation time of the $\mathrm{BTZ} \mathrm{BH}$ is obtained as

$$
t_{\text {evaporation }}=-\frac{l^{2}}{M} .
$$

After that we calculate the emission rate of the BekensteinHawking particles

$$
\Gamma=\frac{M^{3 / 2}}{l} .
$$

The total number of the outgoing Bekenstein-Hawking particles is obtained by following relation $[75,76]$ :

$$
\frac{d N}{d M}=\frac{\Gamma}{d M / d t}=-\frac{l}{\sqrt{M}},
$$

and it is found as

$$
N=-2 l \sqrt{M}
$$

Note that it does not depend on the spin of the particles so both vector and scalar particles radiate from $\mathrm{BBH}$ with the same number of particles $[75,76]$.

For the case of scalar particles with the effect of quantum gravity, the total number of the outgoing BekensteinHawking particles is

$$
N=\frac{-2 l \sqrt{M}}{\sqrt{\left(1-2 \alpha m_{p}^{2}\right)}} .
$$

The total number of Bekenstein-Hawking particles $(N)$ increases with the effect of the quantum gravity constant $\alpha$. However, at some point, $N$ becomes zero, and no particles are emitted. The results of the calculation of the total number of tunneling massless Bekenstein-Hawking particles are compatible with recent studies on the information paradox [75]. Also, one can calculate the number of gravitons in the $\mathrm{BH}$ quantum $N$ portrait with similar conclusions $[56,78,79]$. In this paper, we follow the same proposal as [75], but we investigate it differently for $2+1$ dimensions, imagining a $\mathrm{BH}$ to be a Bose-Einstein condensate with very large and massive gravitons. To solve the information paradox, BHR is thought of as resulting from a decrease in the condensate with nonthermal properties of order $1 / N$ when a two-body interaction occurs and also gives evidence of a quantum $N$ portrait of a semiclassical $\mathrm{BH}[78,79]$. One can interpret each Bekenstein-Hawking radiated particle as an information storage unit; in fact, there is an expected link between the entropy and the particle number, so that the BHR can be thought of as sparse in a semiclassical (corpuscular) regime.

\section{Conclusions}

In this paper, first by using the generalized Klein-Gordon and Proca equations, we investigate the scalar/vector particles tunneling from a $\mathrm{BBH}$ and recover the corresponding Bekenstein-Hawking temperatures. For this purpose, first we use the Proca equation for the tunneling spin-1 (vector) particles on the background of $\mathrm{BBH}$ space-time. Using the
WKB approximation to the Proca equation, we find the set of field equations. Then we use the Hamilton-Jacobi ansatz to solve these equations. To solve these equations, we take the determinant of coefficient matrix as zero and we expand the functions $f(r)$ in Taylor's series near horizon to find the radial wave equation $W(r)$ using the complex path integral. Then we use the surface gravity and calculate the probability of tunneling of spin-1 particles from the BBH. Using the Boltzmann formula, we derive the corresponding BekensteinHawking temperature. It is worthwhile to mention here that the self- gravitating effects have been neglected; however there are back-reaction effects because of the BBH geometry. Moreover, we calculate the Bekenstein-Hawking temperature only in a leading order term. Hence, we can conclude that Bekenstein-Hawking temperature is not dependent on the types of particles and also the tunneling probabilities are same. Therefore, their corresponding Bekenstein-Hawking temperatures are the same for all kinds of particles.

Second, we use the effect of GUP that the existence of a minimal length leads to the modification of the Heisenberg uncertainty principle on the tunneling scalar particles. The GUP contains an additional quadratic term in momentum in addition to a minimal length. After we generalize the Klein-Gordon equation using the effect of the GUP, we focus on the Hamilton-Jacobi method to determine the tunneling probability of the scalar particles. Again we use the WKB approximation and Hamilton-Jacobi ansatz in the tunneling formalism and calculate the imaginary part of the action in order to obtain the Bekenstein-Hawking temperature. Hence, it is shown that if the GUP is used, then the BekensteinHawking temperature of the tunneling scalar particle at the event horizon differs from the original case and the Bekenstein-Hawking temperature has a nonthermal feature. The back-reactions on the black hole have also similar nonthermal effects on the Bekenstein-Hawking temperature. It is concluded that using the GUP decreases the backreacted effects on the Bekenstein-Hawking temperature. However, the GUP effects are not sufficient to extinguish the Bekenstein-Hawking temperature.

Scalar and vector particles radiate from a $\mathrm{BBH}$ with equivalent energies if the GUP is not used. After one uses the generalized Klein-Gordon equation with the effect of GUP, the corrected temperature decreases with the effects of quantum gravity, and at some point, remnants are left. Then, we calculate the total number of emitted BekensteinHawking particles from the special case of a $\mathrm{BBH}$, which is a BTZ BH. Also, we check the effects of quantum gravity on the total number of emitted particles from the $\mathrm{BH}$. It is shown that the emitted Bekenstein-Hawking particles are information-carrying units. This indicates a corpuscular interpretation instead of an undulatory one, and when the $\mathrm{BH}$ collapses, its unitary property is preserved, and the $\mathrm{BH}$ evaporates. Hence, the effect of quantum gravity balances the classical tendency of rising temperature, and there exist remnants. In summary, it is very rare for the BHR to be extremely diluted. The BHR particles are discrete during propagation and also appear as particles later on so that the $\mathrm{BHR}$ particles appear to be a particle feature. 


\section{Conflicts of Interest}

The author declares that there are no conflicts of interest regarding the publication of this paper.

\section{Acknowledgments}

This work was supported by the Chilean FONDECYT Grant no. 3170035 .

\section{References}

[1] J. D. Bekenstein, "The quantum mass spectrum of the Kerr black hole," Lettere Al Nuovo Cimento Series 2, vol. 11, no. 9, pp. 467470, 1974.

[2] J. D. Bekenstein, "Black holes and entropy," Physical Review. D. Particles and Fields. Third Series, vol. 7, pp. 2333-2346, 1973.

[3] J. D. Bekenstein, "Generalized second law of thermodynamics in black-hole physics," Physical Review D, vol. 9, no. 12, pp. 32923300, 1974.

[4] S. W. Hawking, "Particle creation by black holes," Communications in Mathematical Physics, vol. 43, no. 3, pp. 199-220, 1975.

[5] G. W. Gibbons and S. W. Hawking, "Cosmological event horizons, thermodynamics, and particle creation," Physical Review. D. Particles and Fields. Third Series, vol. 15, no. 10, pp. 2738-2751, 1977.

[6] P. Kraus and F. Wilczek, "Some applications of a simple stationary line element for the Schwarzschild geometry," Modern Physics Letters A. Particles and Fields, Gravitation, Cosmology, Nuclear Physics, vol. 9, no. 40, pp. 3713-3719, 1994.

[7] P. Kraus and F. Wilczek, "Effect of self-interaction on charged black hole radiance," Nuclear Physics B, vol. 437, no. 1, pp. 231242, 1995.

[8] M. K. Parikh and F. Wilczek, "Hawking radiation as tunneling," Physical Review Letters, vol. 85, no. 24, pp. 5042-5045, 2000.

[9] M. K. Parikh, "New coordinates for de Sitter space and de Sitter radiation," Physics Letters B, vol. 546, no. 3-4, pp. 189-195, 2002.

[10] M. Parikh, "A secret tunnel through the horizon," International Journal of Modern Physics D, vol. 13, no. 10, pp. 2351-2354, 2004.

[11] M. Angheben, M. Nadalini, L. Vanzo, and S. Zerbini, "Hawking radiation as tunneling for extremal and rotating black holes," Journal of High Energy Physics, vol. 2005, no. 5, article 014, 2005.

[12] K. Srinivasan and T. Padmanabhan, "Particle production and complex path analysis," Physical Review D, vol. 60, no. 2, Article ID 024007, 20 pages, 1999.

[13] S. Shankaranarayanan, K. Srinivasan, and T. Padmanabhan, "Method of complex paths and general covariance of Hawking radiation," Modern Physics Letters A. Particles and Fields, Gravitation, Cosmology, Nuclear Physics, vol. 16, no. 9, pp. 571-578, 2001.

[14] J. Ahmed and K. Saifullah, "Hawking radiation of Dirac particles from black strings," Journal of Cosmology and Astroparticle Physics, vol. 2011, no. 8, 2011.

[15] I. Sakalli and A. Ovgun, "Uninformed Hawking radiation," EPL, vol. 110, no. 1, Article ID 10008, 2015.

[16] K. Jusufi, "Scalar particles emission from black holes with topological defects using Hamilton-Jacobi method," Astrophysics and Space Science, vol. 360, p. 22, 2015.

[17] L. Vanzo, G. Acquaviva, and R. Di Criscienzo, "Tunnelling methods and Hawking's radiation: achievements and prospects,"
Classical and Quantum Gravity, vol. 28, no. 18, Article ID 183001, 183001, 80 pages, 2011.

[18] R. Kerner and R. B. Mann, "Tunnelling, temperature, and TaubNUT black holes," Physical Review D, vol. 73, no. 10, Article ID 104010, 2006.

[19] R. Kerner and R. B. Mann, "Fermions tunnelling from black holes," Classical and Quantum Gravity, vol. 25, no. 9, Article ID 095014, 2008.

[20] R. Kerner and R. B. Mann, "Charged fermions tunnelling from kerr-newman black holes," Physics Letters B, vol. 665, no. 4, pp. 277-283, 2008.

[21] A. Yale and R. B. Mann, "Gravitinos tunneling from black holes," Physics Letters. B. Particle Physics, Nuclear Physics and Cosmology, vol. 673, no. 2, pp. 168-172, 2009.

[22] I. Sakalli and A. Ovgun, "Gravitinos tunneling from traversable Lorentzian wormholes," Astrophysics and Space Science, vol. 359, no. 1, article no. 32, 2015.

[23] S. I. Kruglov, "Black hole radiation of spin-1 particles in $(1+2)$ dimensions," Modern Physics Letters A, vol. 29, no. 39, Article ID 1450203, 2014.

[24] S. I. Kruglov, "Black hole emission of vector particles in $(1+1)$ dimensions," International Journal of Modern Physics A. Particles and Fields. Gravitation. Cosmology, vol. 29, no. 22, 1450118, 10 pages, 2014.

[25] I. Sakalli and A. Ovgun, "Hawking radiation of spin-1 particles from a three-dimensional rotating hairy black hole," Journal of Experimental and Theoretical Physics, vol. 121, no. 3, pp. 404407, 2015.

[26] I. Sakalli and A. Ovgun, "Quantum tunneling of massive spin1 particles from non-stationary metrics," General Relativity and Gravitation, vol. 48, no. 1, article 1, 10 pages, 2016.

[27] I. Sakalli and A. Ovgun, "Tunnelling of vector particles from Lorentzian wormholes in $3+1$ dimensions," European Physical Journal Plus, vol. 130, no. 6, article no. 110, 2015.

[28] R. Li and J. Zhao, "Hawking radiation of massive vector particles from the linear dilaton black holes," The European Physical Journal Plus, vol. 131, p. 249, 2016.

[29] G. Li and J. Mo, "Tunneling radiation as new perspective of understanding the thermodynamics in $f(R)$ gravity," Astrophysics and Space Science, vol. 361, p. 251, 2016.

[30] T. Ibungochouba Singh, I. Ablu Meitei, and K. Yugindro Singh, "Hawking radiation as tunneling of vector particles from KerrNewman black hole," Astrophysics and Space Science. An International Journal of Astronomy, Astrophysics and Space Science, vol. 361, no. 3, Paper No. 103, 7 pages, 2016.

[31] B. Chen, "Tunneling radiation of vector particles in four and five dimensional black holes," International Journal of Theoretical Physics, vol. 55, no. 7, pp. 3181-3190, 2016.

[32] Z. Feng, G. Li, and P. Jiang, "Deformed Hamilton-Jacobi Equations and the Tunneling Radiation of the Higher-Dimensional $\mathrm{RN}-(\mathrm{A}) \mathrm{dS}$ Black Hole," International Journal of Theoretical Physics, vol. 55, no. 7, pp. 3079-3087, 2016.

[33] I. Sakalli, M. Halilsoy, and H. Pasaoglu, "Fading Hawking radiation," Astrophysics and Space Science, vol. 340, no. 1, pp. 155160, 2015.

[34] I. Sakalli and A. Ovgun, "GUP assisted Hawking radiation of rotating acoustic black holes," Astrophysics and Space Science, vol. 361, no. 10, article 330, 2016.

[35] I. Sakalli and A. Ovgun, "Black hole radiation of massive spin-2 particles in $(3+1)$ dimensions," The European Physical Journal Plus, vol. 131, no. 6, article 184, 2016. 
[36] I. Sakalli and A. Ovgun, "Hawking Radiation of Mass Generating Particles from Dyonic Reissner-Nordström Black Hole," Journal of Astrophysics and Astronomy, vol. 37, no. 3, article 21, 2016.

[37] K. Jusufi and A. Ovgun, "Tunneling of massive vector particles from rotating charged black strings," Astrophysics and Space Science, vol. 361, no. 7, article 207, 2016.

[38] A. Ovgun and K. Jusufi, "Massive vector particles tunneling from noncommutative charged black holes and their GUPcorrected thermodynamics," The European Physical Journal Plus, vol. 131, no. 5, article 177, 2016.

[39] I. Sakalli, A. Ovgun, and K. Jusuf, "GUP assisted Hawking radiation of rotating acoustic black holes," Astrophysics and Space Science. An International Journal of Astronomy, Astrophysics and Space Science, vol. 361, no. 10, article 330, 2016.

[40] K. Jusufi, "Dirac particles tunneling from black holes with topological defects," General Relativity and Gravitation, vol. 48, no. 8, article 105, 12 pages, 2016.

[41] K. Jusufi, "Scalar particles emission from black holes with topological defects using Hamilton-Jacobi method," Astrophysics and Space Science, vol. 360, no. 1, article 22, 2015.

[42] K. Jusufi, "Hawking radiation via tunneling from the spacetime of a spinning cosmic string black holes," General Relativity and Gravitation, vol. 47, no. 10, article 124, 2015.

[43] G. Chen and Y. Huang, "Hawking radiation of vector particles as tunneling from the apparent horizon of Vaidya black holes," International Journal of Modern Physics A, vol. 30, no. 15, 2015.

[44] G. Chen, S. Zhou, and Y. Huang, Astrophys. Space Sci, vol. 357, 51, 2015.

[45] A. Ovgun, "Entangled Particles Tunneling From a Schwarzschild Black Hole immersed in an Electromagnetic Universe with GUP," International Journal of Theoretical Physics, vol. 55, no. 6, pp. 2919-2927, 2016.

[46] V. Akhmedova, T. Pilling, A. de Gill, and D. Singleton, "Temporal contribution to gravitational WKB-like calculations," Physics Letters. B. Particle Physics, Nuclear Physics and Cosmology, vol. 666, no. 3, pp. 269-271, 2008.

[47] E. T. Akhmedov, T. Pilling, and D. Singleton, "Subtleties in the quasi-classical calculation of Hawking radiation," International Journal of Modern Physics. D. Gravitation, Astrophysics, Cosmology, vol. 17, no. 13-14, pp. 2453-2458, 2008.

[48] V. Akhmedova, T. Pilling, A. de Gill, and D. Singleton, "Comments on anomaly versus $\mathrm{WKB} /$ tunneling methods for calculating Unruh radiation," Physics Letters. B. Particle Physics, Nuclear Physics and Cosmology, vol. 673, no. 3, pp. 227-231, 2009.

[49] E. T. Akhmedov, V. Akhmedova, and D. Singleton, "Hawking temperature in the tunneling picture," Physics Letters B, vol. 642, no. 1-2, pp. 124-128, 2006.

[50] T. Akhmedov, "Thermal radiation of various gravitational backgrounds," International Journal of Modern Physics A, vol. 22, no. 8-9, pp. 1705-1715, 2007.

[51] B. D. Chowdhury, "Problems with tunneling of thin shells from black holes," Pramana, vol. 70, no. 1, pp. 3-26, 2008.

[52] M. H. Ali and K. Sultana, "Tunneling and Temperature of Demiański-Newman Black Holes," International Journal of Theoretical Physics, vol. 53, no. 11, pp. 4035-4048, 2014.

[53] M. A. Rahman and M. H. Ali, "Hawking non-thermal and thermal radiations of Schwarzschild anti-de Sitter black hole by Hamilton-Jacobi method," Astrophysics and Space Science, vol. 345, no. 2, pp. 325-330, 2013.
[54] C. Martinez and J. Zanelli, "Back reaction of a conformal field on a three-dimensional black hole," Physical Review. D. Third Series, vol. 55, no. 6, pp. 3642-3646, 1997.

[55] A. Paul and B. R. Majhi, "Hawking evaporation cascade in the presence of backreaction effect," International Journal of Modern Physics A, vol. 32, no. 16, Article ID 1750088, 2017.

[56] F. Gray, S. Schuster, A. Van-Brunt, and M. Visser, "The Hawking cascade from a black hole is extremely sparse," Classical and Quantum Gravity, vol. 33, no. 11, Article ID 115003, 2016.

[57] J. D. Bekenstein and V. F. Mukhanov, "Spectroscopy of the quantum black hole," Physics Letters. B. Particle Physics, Nuclear Physics and Cosmology, vol. 360, no. 1-2, pp. 7-12, 1995.

[58] J. Makela, "Black hole spectrum: continuous or discrete?" Physics Letters B, vol. 390, no. 1-4, pp. 115-118, 1997.

[59] S. Hod, "Discrete black-hole radiation and the information loss paradox," Physics Letters A, no. 2-3, pp. 144-148, 2002.

[60] S. Hod, "The Hawking cascades of gravitons from higherdimensional Schwarzschild black holes," Physics Letters B, vol. 756, pp. 133-136, 2016.

[61] M. Banados, C. Teitelboim, and J. Zanelli, "Black hole in threedimensional spacetime," Physical Review Letters, vol. 69, no. 13, pp. 1849-1851, 1992.

[62] L. Mersini-Houghton, "Backreaction of Hawking radiation on a gravitationally collapsing star I: Black holes?” Physics Letters, Section B: Nuclear, Elementary Particle and High-Energy Physics, vol. 738, pp. 61-67, 2014.

[63] R. A. Konoplya, "Influence of the back reaction of the Hawking radiation upon black hole quasinormal modes," Physical Review D, vol. 70, Article ID 047503, 2004.

[64] A. Paul and B. R. Majhi, "Hawking evaporation cascade in the presence of backreaction effect," International Journal of Modern Physics A, vol. 32, no. 16, 11 pages, 2017.

[65] I. L. Sofronov, L. Dovgilovich, and N. Krasnov, "Application of transparent boundary conditions to high-order finitedifference schemes for the wave equation in waveguides," Applied Numerical Mathematics. An IMACS Journal, vol. 93, pp. 195-205, 2015.

[66] M. Maggiore, "A generalized uncertainty principle in quantum gravity," Physics Letters B, vol. 304, pp. 65-69, 1993.

[67] R. J. Adler, P. Chen, and D. I. Santiago, "The generalized uncertainty principle and black hole remnants," General Relativity and Gravitation, vol. 33, no. 12, pp. 2101-2108 (2002), 2001.

[68] A. Kempf, G. Mangano, and R. B. Mann, "Hilbert space representation of the minimal length uncertainty relation," Physical Review D, vol. 52, no. 2, pp. 1108-1118, 1995.

[69] M. Faizal and M. M. Khalil, "GUP-corrected thermodynamics for all black objects and the existence of remnants," International Journal of Modern Physics A, vol. 30, no. 22, Article ID 1550144, 2015.

[70] M. A. Anacleto, F. A. Brito, and E. Passos, "Quantum-corrected self-dual black hole entropy in tunneling formalism with GUP," Physics Letters B, vol. 749, pp. 181-186, 2015.

[71] X. Li and G. Chen, "Massive vector particles tunneling from Kerr and Kerr-Newman black holes," Physics Letters B, vol. 751, pp. 34-38, 2015.

[72] M. A. Anacleto, F. A. Brito, G. C. Luna, E. Passos, and J. Spinelly, "Quantum-corrected finite entropy of noncommutative acoustic black holes," Annals of Physics, vol. 362, pp. 436-448, 2015.

[73] A. F. Ali, S. Das, and E. C. Vagenas, "Discreteness of space from the generalized uncertainty principle," Physics Letters. B, vol. 678, no. 5, pp. 497-499, 2009. 
[74] X.-Q. Li, "Massive vector particles tunneling from black holes influenced by the generalized uncertainty principle," Physics Letters B, vol. 763, pp. 80-86, 2016.

[75] W. Muck, "Behaviour of charged collapsing fluids after hydrostatic equilibrium in $R^{n}$ gravity," The European Physical Journal C, vol. 77, article 374, 2016.

[76] A. Alonso-Serrano and M. Visser, "Entropy/information flux in Hawking radiation," https://arxiv.org/abs/1512.01890.

[77] V. P. Frolov and A. Zelnikov, "Introduction to Black Hole Physics," Introduction to Black Hole Physics, pp. 1-504, 2012.

[78] G. Dvali and C. Gomez, "Black hole's quantum $N$ portrait," Fortschritte der Physik. Progress of Physics, vol. 61, no. 7-8, pp. 742-767, 2013.

[79] G. Dvali and C. Gomez, "Black holes as critical point of quantum phase transition," The European Physical Journal C, vol. 74, no. 2, Article ID 2752, pp. 1-12, 2014. 

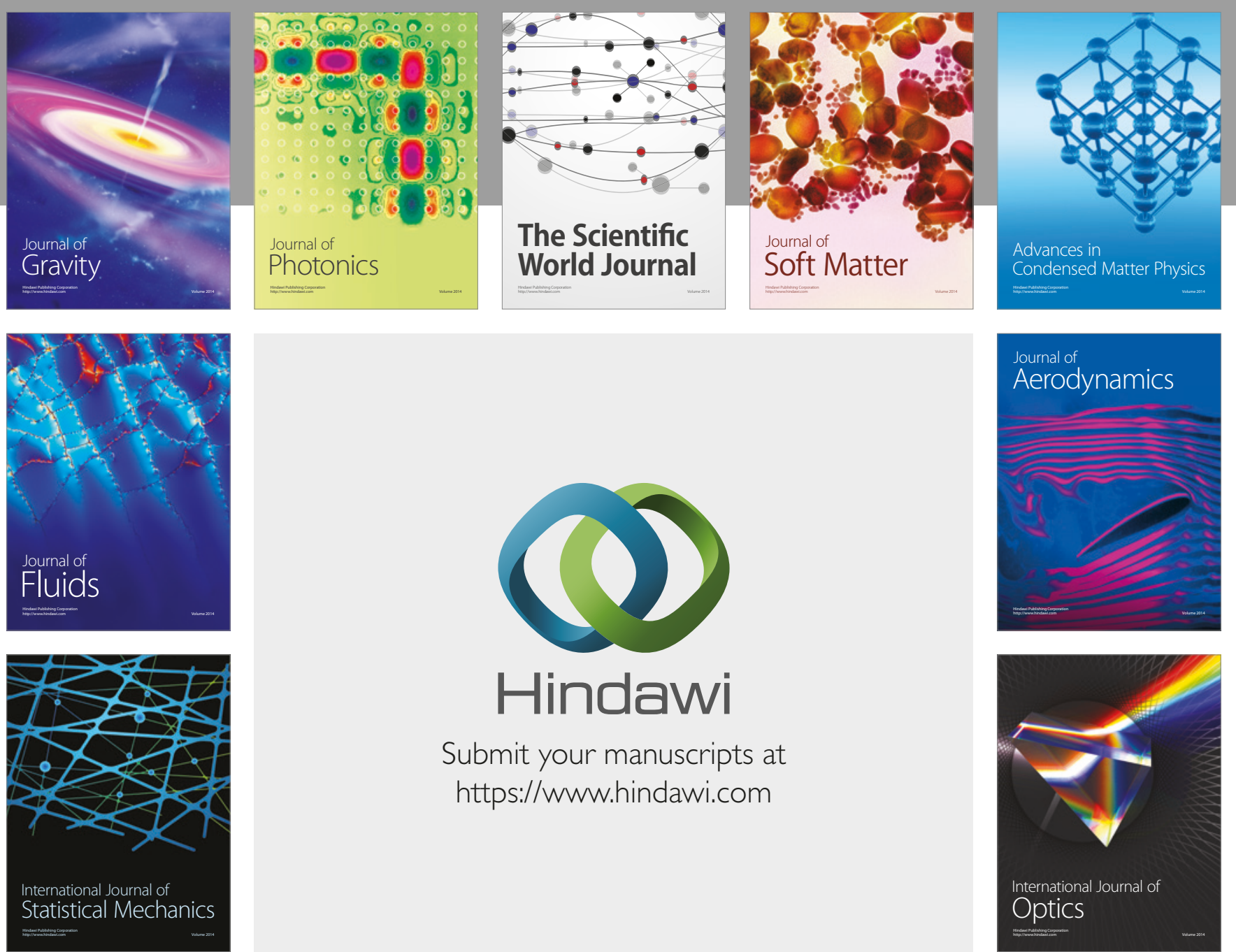

Submit your manuscripts at

https://www.hindawi.com
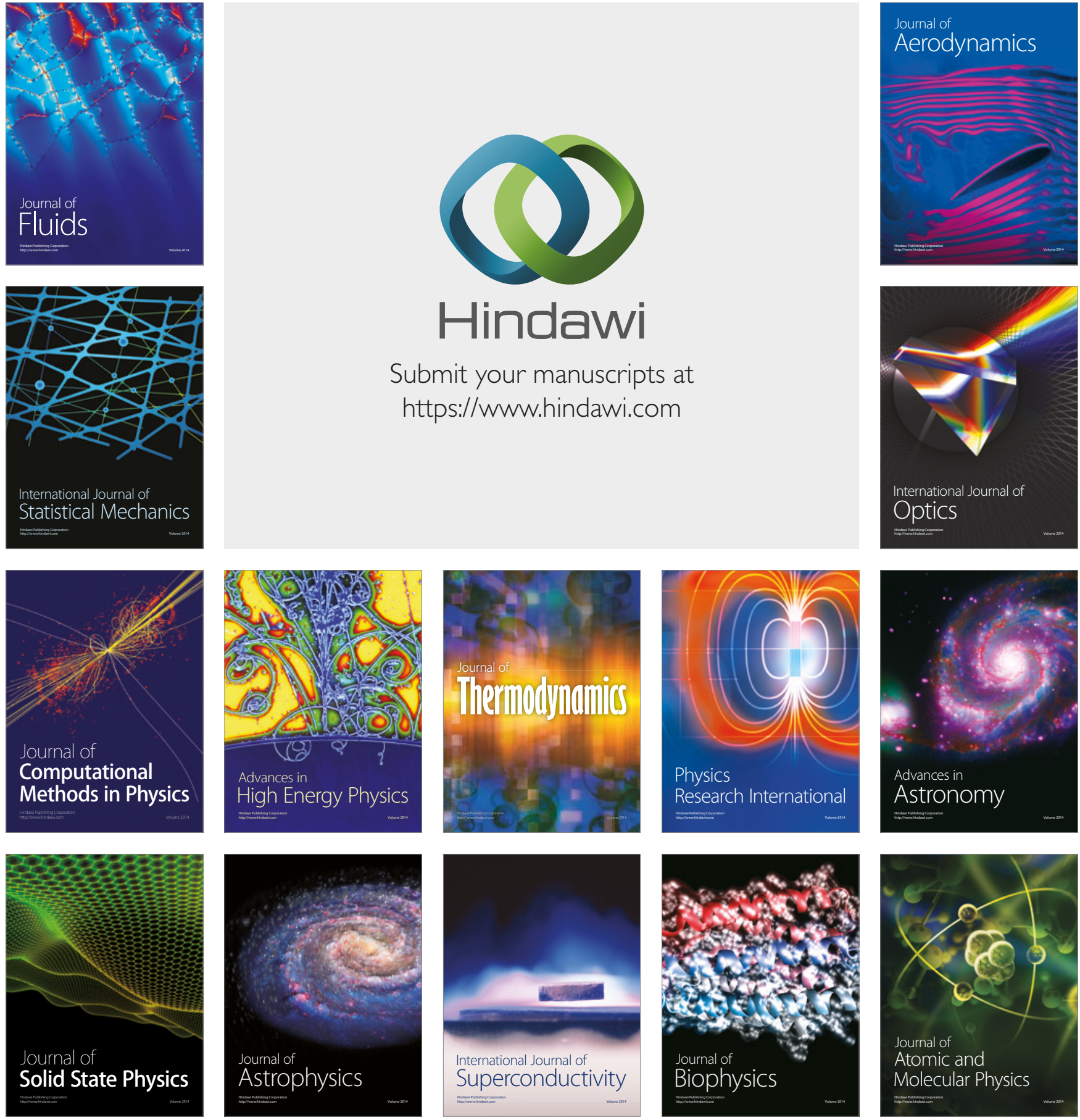